\title{
Diabetes and neurology: hemichorea-hemiballism in hyperglycaemia
}

\author{
Authors: Kim Yen Galloway, ${ }^{\mathrm{A}}$ Osman Younus, ${ }^{\mathrm{A}}$ Graziella Quattrocchi, ${ }^{\mathrm{A}}$ Bazo Raheem, ${ }^{\mathrm{A}}$ George Dervenoulas ${ }^{\mathrm{A}}$ and Eli Silber ${ }^{\mathrm{A}}$
}

\section{Introduction}

Diabetes affects a large proportion of the population, with the incidence rising. It also has a wide range of complications. We describe here an uncommon neurological complication of poor diabetic control. Prompt recognition of this condition is essential to improve the outcome.

\section{Case presentation}

A 71-year-old Chinese man with longstanding diabetes presented with a 4-day history of progressively worsening uncontrollable continuous non-rhythmic right upper limb movements which resolved during sleep. One week prior, his general practitioner noted poor glycaemic control and started him on sitagliptin. Two days later, he reported right shoulder tingling, gradually spreading down the right arm. This was followed, a few days later, by right hand involuntary movements, gradually affecting his entire right upper limb. The patient visited a Chinese medicine practitioner who performed acupuncture and cupping, eventually presenting to hospital after no improvement.

His past medical history included hypertension, hypothyroidism and type 2 diabetes mellitus with poor control, partially due to refusal of insulin because of needle phobia. His regular medications included metformin $500 \mathrm{mg}$ bd, sitagliptin $25 \mathrm{mg}$ od, amlodipine and levothyroxine.

Neurological examination showed right upper and lower limb involuntary movements consistent with hemichoreahemiballism (HCHB). Blood tests including full blood count, coagulation, liver and kidney function, bone profile and C-reactive protein (CRP) were normal. His blood sugar was $42.4 \mathrm{mmol} / \mathrm{L}$ (range 3.0-7.7) with ketones of $0.8 \mathrm{mmol} / \mathrm{L}$ (0.6-1.5) and serum osmolality of $297 \mathrm{mOsm} / \mathrm{kg}$ (275-295) with Osm gap $12.0 \mathrm{mOsm} / \mathrm{kg}(<10)$. His $\mathrm{HbA}_{1 \mathrm{c}}$ was $141 \mathrm{mmol} /$ mol (20-41).

Computed tomography (CT) of the head showed unilateral left striatum faint hyperdensity, with sparing of the internal capsule and with no mass effect, suggestive of HCHB due to hyperglycaemia. Subsequent magnetic resonance imaging (MRI) of the brain was normal.

Intensive glycaemic control was started with insulin. He was treated with clonazepam $500 \mu \mathrm{g}$ three times a day (tds), to be uptitrated to $1 \mathrm{mg}$ tds. He improved and was discharged, with diabetic clinic follow-up and plan to start risperidone.

\section{Discussion}

HCHB presents as continuous involuntary high-amplitude movements affecting one side of the body. $\mathrm{HCHB}$ is a rare syndrome with a prevalence of less than 1/100,000; the majority of individuals affected are Asian women in their 7 th decade. ${ }^{1}$ The increased incidence in Asian populations suggests a genetic predisposition. ${ }^{2}$ Ischaemic/haemorrhagic stroke is the most common cause of $\mathrm{HCHB}$, followed by non-ketotic hyperglycaemia. Other aetiologies are shown in Table $1 .^{3}$

The pathophysiology of HCHB due to hyperglycaemia (also known as C-H-BG: chorea, hyperglycaemia, basal ganglia syndrome) is still uncertain. One suggested mechanism is disruption of the blood-brain barrier (BBB) and transient ischaemia of vulnerable neurons caused by hyperviscosityrelated hyperglycaemia. Additionally, hyperglycaemia may also impair cerebral autoregulation, causing anaerobic metabolism activation and depletion of gamma-aminobutyric acid (GABA), the main striatal inhibitory neurotransmitter. ${ }^{2}$ Interestingly, HBHC may present a few weeks after blood glucose levels are controlled, suggesting a delayed reaction to severe hyperglycaemia.

The majority of described cases have a good prognosis. In conclusion, a low threshold in screening for hyperglycaemia in $\mathrm{HCHB}$, even when there is no known history of diabetes, ${ }^{4}$ is essential, as prompt diagnosis and management may significantly improve the outcome.

\section{Table 1. Less common causes of hemichorea-}

\section{hemiballism}

\section{Primary/inherited}

Wilson's disease

Multiple sclerosis

Huntington's disease

Motor neurone disease

Demyelinating disease

\section{Secondary/acquired} Vitamin B12 deficiency

Vascular

Autoimmune

Endocrine

Drugs

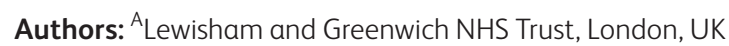




\section{Kim Yen Galloway, Osman Younus, Graziella Quattrocchi et al}

\section{Conflicts of interest}

None declared.

\section{References}

1 Jaafar J, Rahman RA, Draman N et al. Hemiballismus in uncontrolled diabetes mellitus. Korean J Fam Med 2018;399:200-3.
2 Bizet J, Cooper CJ, Quansah R et al. Chorea, hyperglycaemia, basal ganglia syndrome (C-H-BG) in an uncontrolled diabetic patient with normal glucose levels at presentation. Am J Case Rep 2014;15:143-6.

3 Fielder E, Biller J. Case report: Hemichorea in a woman with diabetes. Pract Neurol 2019;19:44-6.

4 Pinsker JE, Shalileh K, Rooks VJ, Pinsker RW. Hemichoreahemiballism secondary to non-ketotic hyperglycemia. J Clin Med Res 2015;7:729-30. 